\title{
Role of Inflammatory Cytokines, Growth Factors and Adipokines in Adipogenesis and Insulin Resistance
}

\author{
Layla Al-Mansoori ${ }^{1}$, Hend Al-Jaber ${ }^{1}$, Mohammad Shoaib Prince ${ }^{2}$ and \\ Mohamed A. Elrayess ${ }^{1,3}$ (D)
}

Received 9 July 2021; accepted 31 August 2021

\begin{abstract}
Obesity, manifested by increased adiposity, represents a main cause of morbidity in the developed countries, causing increased risk of insulin resistance and type 2 diabetes mellitus. Recruitment of macrophages and activation of innate immunity represent the initial insult, which can be further exacerbated through secretion of chemokines and adipocytokines from activated macrophages and other cells within the adipose tissue. These events can impact adipogenesis, causing dysfunction of the adipose tissue and increased risk of insulin resistance. Various factors mediate adiposity and related insulin resistance including inflammatory and non-inflammatory factors such as pro and anti-inflammatory cytokines, adipokines and growth factors. In this review we will discuss the role of these factors in adipogenesis and development of insulin resistance and type 2 diabetes mellitus in the context of obesity. Understanding the molecular mechanisms that mediate adipogenesis and insulin resistance could help the development of novel therapeutic strategies for individuals at higher risk of insulin resistance and type 2 diabetes mellitus.
\end{abstract}

Key Words: cytokines; adipokines; growth factors; adipogenesis; IR.

\section{INTRODUCTION}

Obesity represents the fourth most frequent cause of morbidity in the developed countries according to the world health organization (WHO) reports [1,2]. As obesity becomes more prevalent, the global threat of diabetes, particularly type 2 diabetes mellitus (T2DM), is increasing. It is estimated that more than 500 million people are expected to develop T2DM by 2030 [1, 3, 4]. Although

\footnotetext{
${ }^{1}$ Biomedical Research Center, Qatar University, Doha, Qatar ${ }^{2}$ Department of Sports and Wellness, College of North Atlantic Qatar (CNAQ), Doha, Qatar

${ }^{3}$ To whom correspondence should be addressed at Biomedical Research Center · Qatar University · Doha, Qatar. Email: m.elrayess@qu.edu.qa
}

several factors contribute to the increased incidents of diabetes, excess body fat [2] and abdominal obesity [5] are thought to constitute the most important risk factors for the development of T2DM. These risk factors have been directly linked to decreased physical activity due to changes in lifestyle, and increased consumption of food containing high fat $[2,5,6]$. At the molecular level, two major factors are associated with obesity-induced T2DM: insulin resistance (IR) [7] and inflammation [8-10]. People with T2DM usually manifest highly active immune response with increased levels of inflammatory factors in their bodies. In early 1990s, tumor necrosis factor alpha $(\mathrm{TNF} \alpha)$ was found to be the major inflammatory factor secreted by the immune cells. Later other cytokines were found to play an important role in suppressing insulin 
signaling pathway and producing IR [11]. Additional factors with no direct inflammatory effect were also found to increase the risk of IR and T2DM. These included various growth factors and adipokines $[12,13]$. One of the underlying mechanisms linking these factors with IR in obesity is the process of adipogenesis that involves generating fat cells from their precursors $[10,14]$. In this review we will discuss the role of inflammatory cytokines, growth factors and adipokines in adipogenesis and development of IR and T2DM in obesity.

\section{Adipogenesis and Adipose Tissue Formation}

Adipocytes (fat cells) make up the majority of the adipose tissue, although the latter also contains preadipocytes (fat stem cells), macrophages, fibroblasts, blood cells, and endothelial cells [3, 15-17]. Adipose tissue is classified into three categories based on its morphology and metabolic functions, namely white, brown, and beige adipose tissue [18, 19]. Adipose tissue is present in various bodily compartments, with roughly $80 \%$ of total body fat being found under the skin (subcutaneous adipose tissue or SAT) and the remaining 20\% around the digestive organs (mesenteric and omental adipose tissue, or OAT) [20]. Adipose tissue's main function is to store energy in the form of fat (triacylglycerols). However, when the ratio of energy intake exceeds energy expenditure, the number of fat cells (hyperplasia) and/or their size (hypertrophy) rises, leading to obesity (Fig. 1). Adipose tissue also performs a variety of additional functions, including hormone synthesis, adipokines production, and immunological modulation. Metabolism, insulin sensitivity, and immunological function are all influenced by adipose tissue [21].

Several transcription factors regulate adipogenesis throughout late embryonic development and subsequently in adulthood, stimulating the differentiation of mesenchemial stem cells and preadipocytes to produce mature adipocytes [20]. A group of these factors and their effects are described in Fig. 2. Briefly, the process of adipose tissue formation (adipogenesis) involves three distinct phases: The first phase includes the commitment of mesenchymal stem cells (MSCs) into the adipogenic lineage under the influence of adipogenic enhancing signals such as insulin-like growth factor 1 (IGF-1) and insulin itself $[22,23]$. This is followed by cell proliferation known as "mitotic clonal expansion phase". At certain level of expansion, profound changes take place and the cells change from fibroblastic to spherical shape and the preadipocytes start expressing lipogenic genes including peroxisome proliferator-activated receptor- $\gamma(\operatorname{PPAR} \gamma)$, CCAAT/enhancer-binding proteins (C/EBPs) family, CoA carboxylase (ACC) and adipocyte fatty acid binding protein (aP2), triggering adipogenic differentiation and formation of adipocytes [24].

\section{Obesity characterization}

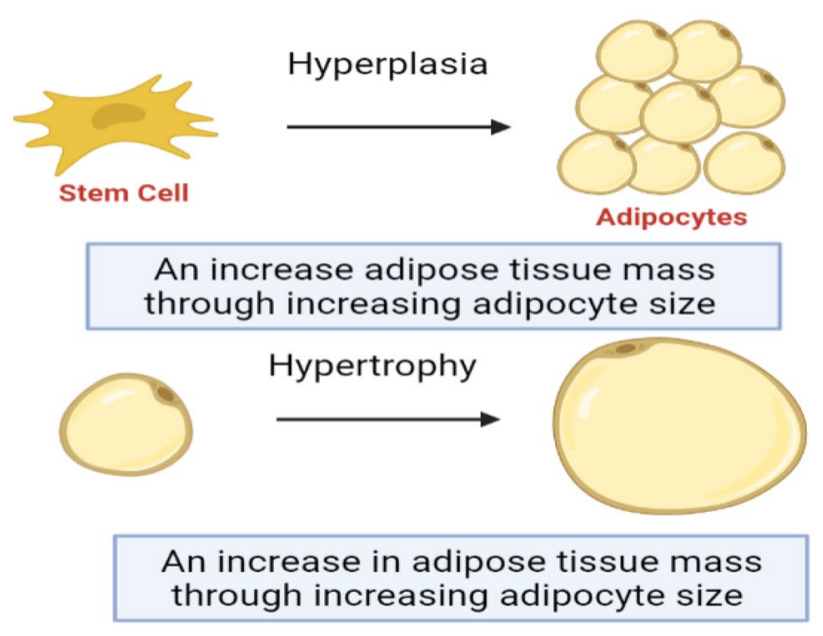

Fig. 1 Obesity-mediated changes in adipocyte numbers (hyperplasia) and size (hypertrophy). 


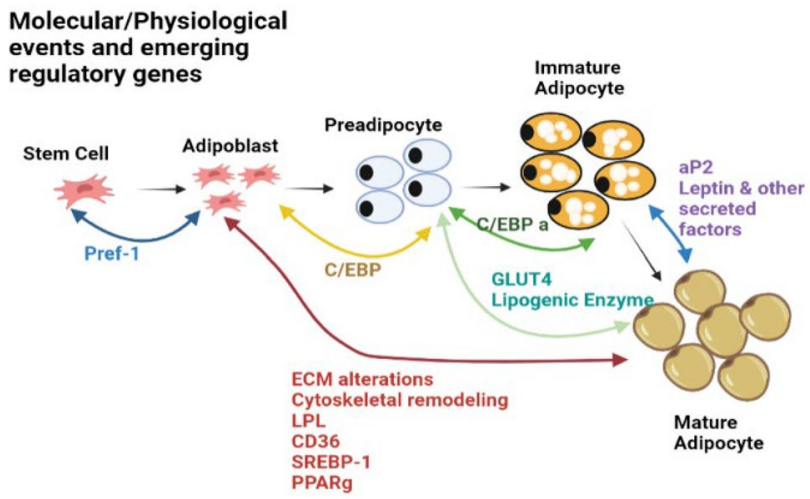

Fig. 2 Factors affecting adipogenesis. Mesenchymal stem cells give rise to adipoblast that further differentiate into preadipocytes under the influence of multiple transcription factors such as preadipocyte factor-1 (Pref-1), sterol regulatory element-binding protein 1 (SREBP-1), peroxisome proliferator-activated receptor gamma (PPAR $\gamma)$ and changes in the extracellular matrix (ECM) and cytoskeleton. Preadipocytes are then further differentiated into immature adipocytes then mature adipocytes under the influence of CCAAT/enhancer-binding protein alpha (C/EBP $\alpha)$, adipocyte protein 2 (aP2), leptin, lipoprotein lipase (LPL), leukocyte differentiation antigen (CD36), and glucose transporter number 4 (GLUT4). The two sided arrows indicate expression of the specific factors throughout the transition period.

\section{Relationship Between Adipogenesis and IR}

IR is a pathological condition that affects insulin metabolic pathways. Liver, muscle, and fat cells lose their ability to respond to insulin. Obesity, hyperglycaemia, and high blood pressure are among the underlying causes of IR in these tissues. Factors such as lifestyle, smoking, and family history may further increase the risk of IR and associated comorbidities such as diabetes, hypertension and cardiovascular disease [25, 26]. Inflammatory cytokines such as plasminogen activator inhibitor 1 , interleukin (IL)-6, IL-8, TNF- $\alpha$, monocyte chemoattractant protein-1 (MCP-1), and leptin are signalling molecules generated by immune cells that regulate IR. TNF- $\alpha$, IL- 6 , and MCP-1 are obesity linked inflammatory cytokines, particularly abdominal obesity. TNF- $\alpha$ and IL- 6 can also trigger IR by inhibiting certain insulin signalling pathways involved in suppressing insulin signal transduction by serine phosphorylation of IRS1 and activation of JAKSTAT signalling pathway, causing a decrease in GLUT4 and IRS1 expression. Moreover, high levels of TNF- $\alpha$ and IL-6 are associated with increased levels of C-reactive protein (CRP), an acute inflammatory marker [27].

Impaired adipogenesis can contribute to the development of IR in target tissues [7]. Some mediators of lipid formation, including protein kinase $\mathrm{C}$ (PKC) and ceramides, can be activated by elevated lipid storage. These active lipid molecules enhance lipid accumulation and induce IR in a variety of target organs [28]. When energy intake increases, the storage capacity of the SAT becomes limited. This triggers the deposition of excess fat around internal tissues and organs, including OAT, skeletal muscles, liver, and heart [29]. Excessive lipid storage causes SAT hypertrophy, which leads to adipose tissue malfunction and increased tissue fibrosis [10]. This triggers further inflammatory processes, lipolysis and IR, leading to T2DM (Fig. 3)[10].

\section{Role of Pro-Inflammatory Cytokines in Adipogenesis and IR}

Inflammation is an adaptive immune response that is triggered by infection as well as tissue or cell injury or damage [30]. Inflammatory factors such as cytokines, chemokines, and vasoactive amines are activated by tissue resident macrophages and mast cells, which in turn trigger the onset of the inflammatory response [31]. Some inflammatory factors have pro-inflammatory properties, whereas others have anti-inflammatory properties. However some of these factors have both proinflammatory and anti-inflammatory actions [30]. The pro and / or anti-inflammatory effects depend on inflammatory condition/situation. After phagocytosis, resident macrophages secrete proinflammatory cytokines that recruit other immune cells and cause acute inflammation. Proinflammatory cytokines enhance inflammation cascade and boost the inflammatory reactions. Some of the known pro-inflammatory cytokines are interleukins (IL-1 $\beta$, 


\section{Adipocyte hypertrophy and Associated Charactersitics}

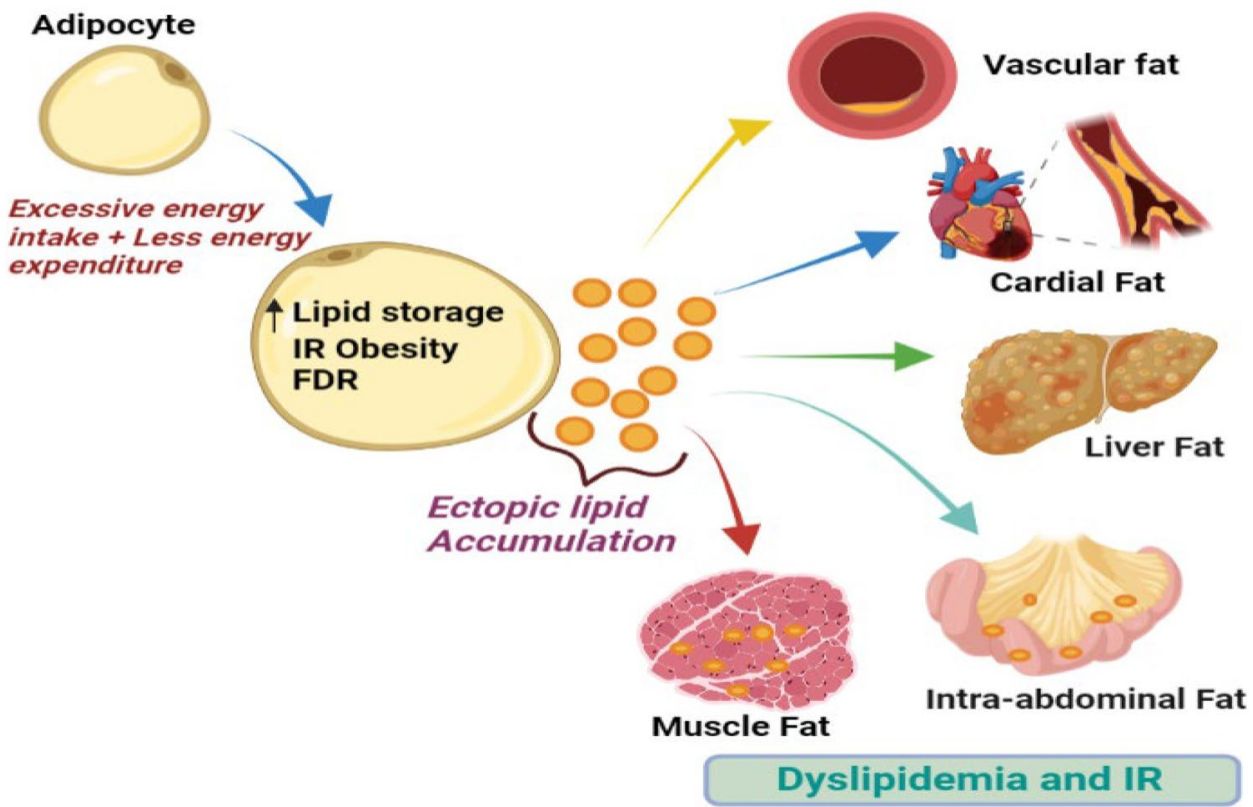

Fig. 3 Adipocyte hypertrophy and associated consequences including ectopic fat deposition and IR (6).

IL-6, IL-7, IL-8, IL-15, IL-17, IL-18, IL-33, IL-34 and IL-1F6), TNF- $\alpha$, oncosatin-M (OSM), interferon (IFN)- $\alpha$, and certain chemokines.

Pro-inflammatory cytokines have been reported to have both inhibitory and stimulatory characteristics on adipogenesis. Among the proinflammatory cytokines, IL-1 $\beta$, IL-6, IL-1F6, IL-15, IL-17, IL-18, IL-33, TNF- $\alpha$, and OSM have been associated negatively with adipogenesis as they impair or reduce adipogenesis. However, other pro-inflammatory cytokines such as IL-7 and IL-34 have been reported to increase adipogenesis (Table 1). Moreover, all of the listed (Table 1) pro-inflammatory cytokines, except for IL-1F6, IL-15, IL-18 and IL-33, induce IR. IL-15, IL-18, IL-33 have been reported to have protective characteristics against IR, while increasing insulin sensitivity. Whereas IL-1F6 has been reported to have no effect on IR. Table 1 lists pro-inflammatory cytokines expressed in adipose tissue, their effect on adipogenesis and association with IR and T2DM.

Among the pro-inflammatory and immunomodulatory cytokines, IL-6 represents one of the most studied factors associated with impaired adipogenesis and IR. IL-6 levels are higher in obese insulin resistant individuals compared to BMI-matched insulin sensitive counterparts [32]. Elevation in IL-6 levels is an indication of obesity related IR and has been positively associated with hyperplasia of adipose tissue [59]. IL-6 also plays an important role in hepatic IR [60] and as a signaling molecule that inhibits adipogenesis [32-34]. Furthermore, IL-6 can act as an immunomodulator in various diseases such as multiple sclerosis and Covid19 infection as indicated recently $[61,62]$. TNF- $\alpha$ is another important player in obesity-associated adipose tissue dysfunction. The anti-adipogenic properties of TNF- $\alpha$ are mediated by the function of its receptor 1 (TNFR1) [63]. Due to increased levels of mitogenactivated protein kinase kinase 4 (MAP4K4), which is involved in TNF- $\alpha$ signaling pathway, the number of preadipocytes undergoing differentiation in the abdominal subcutaneous tissue is reduced, resulting in hypertrophic fat cells in association with obesity. This suggests an inverse relationship between lipid storage and proinflammatory capacity [35-37]. Moreover, reports have indicated that although the lipid storage capacity remains unrestricted by $\mathrm{TNF}-\alpha$ in mature adipocytes, the expression of the insulin signaling intermediates 
Table 1 Pro-inflammatory Cytokines in adipose tissue and their role in adipogenesis and IR

\begin{tabular}{|c|c|c|c|}
\hline $\begin{array}{l}\text { Pro-inflam- } \\
\text { matory } \\
\text { cytokines }\end{array}$ & Expression within the adipose tissue & Effect on adipogenesis & Relation to IR and T2DM \\
\hline IL-6 [32-34] & Preadipocytes, monocytes/macrophages & Impairs adipogenesis & Induces IR \\
\hline $\mathrm{TNF} \alpha[35-37]$ & Monocytes/macrophage, stromal vascular cells, adipocytes & Impairs/inhibits adipogenesis & Induces IR \\
\hline IL-1 $\beta[38-40]$ & Monocytes/macrophage, adipocytes & Impairs adipogenesis & Induces IR \\
\hline IL-33 [41-43] & $\begin{array}{l}\text { Adipocytes, preadipocytes, endothelial cells, fibroblast-like } \\
\text { reticular cells, necrotic cells, cells under stress }\end{array}$ & Reduces/impairs adipogenesis & $\begin{array}{l}\text { Reduces/protects against IR } \\
\text { (increases insulin sensitivity and } \\
\text { tolerance) }\end{array}$ \\
\hline IL-18 [43, 44] & $\begin{array}{l}\text { Stromal vascular cells, macrophages, dendritic cells, epithe- } \\
\text { lial cells, endothelial cells }\end{array}$ & No reported effect & Induces/increases insulin sensitivity \\
\hline IL-15 [45-47] & Adipocytes, stromal vascular cells & Inhibits/impairs adipogenesis & Induces/improves insulin sensitivity \\
\hline IL-34 [48] & Adipocytes, stromal vascular cells & Induces/enhances adipogenesis & Induces IR \\
\hline IL-7 $[49,50]$ & Stromal vascular cells & Induces/enhances adipogenesis & Induces IR \\
\hline $\begin{array}{l}\text { IL-1F6 \& } \\
\text { IL-1F8 [51] }\end{array}$ & Stromal vascular fraction & Impairs adipogenesis & No reported effect \\
\hline $\begin{array}{l}\text { OSM } \\
\text { [52-54] }\end{array}$ & Stromal vascular fraction, macrophages & Inhibits/impairs adipogenesis & Induces IR \\
\hline IL-17 [55-57] & $\mathrm{T}$ helper cells, $\gamma \delta \mathrm{T}$ cells & Inhibits/impairs adipogenesis & Induces IR \\
\hline IFN- $\alpha[58]$ & Fibroblasts, monocytes & Impairs adipogenesis & Induces IR \\
\hline
\end{tabular}

are downregulated, hindering insulin-mediated glucose uptake [63].

Another pro-inflammatory cytokine that plays an important role in obesity-associated impaired adipogenesis and insulin resistance is IL-1 $\beta$. Macrophage-derived IL1- $\beta$ represents an important anti-adipogenic factor that is associated with IR. High concentration of IL1- $\beta$ can inhibit adipocyte differentiation, however it is not the only component of macrophage-derived conditioned medium that induces the anti-adipogenic activity [38-40]. IL1- $\beta$ is upregulated in adipose tissue of obese individuals with IR during the development of IR in adipose cells [64]. IL-33, on the other hand, plays an important protective role during the development of adipose tissue associated inflammation in obesity, although obesity decreases the protective activity of IL-33 in adipocytes [41-43]. Hyperinsulinemia and IR were reduced after treatment with IL-33 [65]. The therapeutic administration of IL-33 leads to several anti-obesity benefits, including the reversal of visceral adipose tissue associated inflammation and reduction of IR [65].

Other interleukins that play important roles in adipogenesis and IR including IL-18, IL-15, IL-34 and IL-7. IL-18 is a key immune response regulator and a pleiotropic proinflammatory cytokine that plays an important role early in the inflammatory cascade process [66]. Phosphorylated Akt is increased by IL-18, while phosphorylated P38 MAPK is downregulated. In obesity and diabetes, higher serum IL-18 levels may be a compensatory response to IR $[43,44]$. IL-15 is another proinflammatory cytokine that directly reduces adipogenesis by upregulating calcineurin [67]. In the absence of IL-15, fat formation in white adipose tissues is reduced, and lipid use is increased by adaptive thermogenesis [45-47]. In addition, IL-15 increases inflammation in adipose tissues, which may contribute to chronic inflammation and obesity-related metabolic syndrome [46]. IL-34 serum concentrations are greatly elevated in obese patients, regardless of their diabetes status. IL-34 levels in the blood are strongly and positively associated with IRrelated metabolic parameters [48]. IL-7 is involved in the induction of adipogenesis and IR in response to a high-fat diet [50]. IL-7 modulates adipose tissue mass through a lymphocyte-independent mechanism, while immune cells involved in white adipose tissue inflammation relay its protective role on glucose homeostasis [50].

In mature adipocytes, IL-1 family member 6 (IL1F6) and IL-1 family member 8 (IL-1F8) can stimulate inflammatory gene expression. IL-1F6 reduces PPAR $\gamma$ expression, which may result in a decreased adipocyte 
development, implying that this cytokine has metabolic effects [51]. Recent data has indicated that OSM is produced by immune cells in white adipose tissue and its levels are dramatically increased in obesity and T2DM [52-54]. OSM has a paracrine effect on adipocytes, generating a proinflammatory phenotype in adipose tissue [68]. By modulating C/EBP activity, OSM slows the initiation of terminal differentiation of adipocytes via the Ras/ERK and STAT5 signaling pathways [53].

IL-17 inhibits the expression of several pro-adipogenic transcription factors, such as PPAR $\gamma$ and C/EBP $\alpha$ [55]. Hence, adipogenesis is suppressed by IL-17 due to the combined action of transcription factors that govern adipocyte differentiation [55-57]. Furthermore, reports have suggested that IL-17 acts as a negative regulator of adipogenesis and glucose metabolism, delaying the onset of obesity [56] similar to other pro-inflammatory cytokines such as IL- $1 \beta$ and TNF- $\alpha$. IFN- $\alpha$ inhibits lipid formation and the expression of adipogenesis-related genes. During early stages of adipogenesis, IFN- $\alpha$ suppresses adipocyte development. Moreover, IFN- $\alpha$ regulates the production of CDK2 and $\mathrm{p} 21$ that stops the cell cycle. Furthermore, IFN- $\alpha$-induced STAT1 phosphorylation inhibits adipocyte development [58].

\section{Role of Anti-Inflammatory Cytokines in Adipogenesis and IR}

In contrast to pro-inflammatory cytokines, antiinflammatory cytokines prevent inflammatory reactions and control the pro-inflammatory cytokines responses. They represent immunoregulatory molecules that control the pro-inflammatory cytokine response by acting in concert with specific cytokine inhibitors and soluble cytokine receptors to regulate the human immune response. Table 2 lists some of the anti-inflammatory cytokines, their expression in adipose tissue and effect on adipogenesis and modulating insulin sensitivity. These include IL-1 receptor antagonist (IL-1Ra), IL-4, IL-5, IL-10, IL-11, IL-13, TGF- $\beta$.

The anti-inflammatory IL-1Ra operates by suppressing the effects of IL-1. Obese individuals have significantly higher serum levels of IL-1Ra [81] that are associated with increased body mass index (BMI) and IR, and is overexpressed in their white adipose tissues [69, 70]. Insulin sensitivity is reduced by IL-1Ra [69], which causes a muscle-specific decrease in glucose absorption. The link between the anti-inflammatory cytokine IL-4 and T2DM was previously established as IL-4 promotes insulin sensitivity, glucose tolerance, and lipid deposit inhibition in order to regulate glucose and lipid metabolism [71-73]. The majority of regulatory cells in lean adipose tissue maintain tissue homeostasis by excreting type 2 cytokines like IL-4, IL-5, and IL-13, which keeps adipose tissue macrophages in an anti-inflammatory condition [82]. Furthermore, IL-5 deficiency causes impairment of eosinophil buildup in the visceral adipose tissues, leading to increased adiposity and IR [74, 75].

The white adipose tissues of obese insulin resistant individuals exhibit elevated IL-10 secretion by proinflammatory macrophages, which causes a negative effect on insulin sensitivity and fat cell metabolism [76]. IL-10 works on IL-10 receptor alpha (IL-R $\alpha$ ) in adipose tissue [83]. However, unlike its role in mice, IL-10 is suggested to have no effect on human adipocyte activity [76]. Studies in mice have shown that IL-10 inhibits diet-induced

Table 2 Anti-inflammatory Cytokines in adipose tissues and their role in adipogenesis and IR

\begin{tabular}{|c|c|c|c|}
\hline $\begin{array}{l}\text { Anti- } \\
\text { inflammatory } \\
\text { cytokines }\end{array}$ & Expression in adipose tissue & Effect on adipogenesis & Relation to IR and T2DM \\
\hline IL-1Ra $[69,70]$ & White adipose tissue & Impairs adipogenesis & Reduces insulin sensitivity \\
\hline IL-4 [71-73] & $\begin{array}{l}\text { Adipocytes, eosinophils, M2-like reparative } \\
\text { macrophages }\end{array}$ & Inhibits lipid deposition & Promotes insulin sensitivity \\
\hline IL-5 $[74,75]$ & Visceral adipose tissue, eosinophils & $\begin{array}{l}\text { Deficiency in Visceral fat promotes adipos- } \\
\text { ity }\end{array}$ & Deficiency in visceral fat promotes IR \\
\hline IL-10 [76, 77] & $\begin{array}{l}\text { Adipocyte progenitors, macrophages, } \\
\text { leukocytes }\end{array}$ & $\begin{array}{l}\text { Deficiency in subcutaneous fat promotes } \\
\text { browning }\end{array}$ & $\begin{array}{l}\text { Promotes IR in adipocytes and insulin } \\
\text { sensitivity in skeletal muscle }\end{array}$ \\
\hline IL-11 [78] & Adipocytes & Inhibits adipogenesis & Not documented \\
\hline IL-13 [79] & Adipocytes & Not documented & Protects against IR \\
\hline TGF- $\beta[80]$ & Adipocytes & Inhibits adipocyte development & Not documented \\
\hline
\end{tabular}


IR by reducing response of macrophages and levels of cytokines in skeletal muscle [84]. Furthermore, energy consumption and adipose thermogenesis are increased in mice missing IL-10. Deletion of IL-10 also protects mice from diet-induced obesity and triggers browning of mouse subcutaneous white adipose tissue. In this model, IL-10 alters chromatin shape as well as C/EBP and activating transcription factor (ATF) occupancy [77].

The anti-inflammatory cytokine IL-11 belongs to the gp130 cytokine co-receptor-related family and is actively produced in differentiating cells in response to PGF $2 \alpha$ stimulation. PGF $2 \alpha$ inhibits adipocyte differentiation via an autocrine negative feedback loop mediated by IL-11, which controls adipogenesis via the STAT1 transcription factor's crucial actions [78]. The anti-inflammatory cytokine IL-13 is produced in the adipose tissue of obese patients, primarily from adipocytes. The I $\mathrm{KB}$ kinase (IKK) stimulates the synthesis of proinflammatory factors in adipocytes, but it also increases the formation of IL-13, which has a specific protective impact by lowering adipose tissue inflammation and IR [79]. Many elements of development, including adipogenesis, are regulated by members of the TGF- $\beta$ superfamily. TGF- $\beta$ and activin A inhibit adipocyte development, offering potential targets for treating human obesity and comorbidities [80].

\section{Role of Growth Factors in Adipogenesis and IR}

Growth factors are biologically active molecules secreted in the body, which can affect cell growth and promote mitosis. They can cause altered gene expression by affecting various signal transduction pathways [85]. Several growth factors that are either protein (over 50 amino acid residues) or peptides (2-50 amino acid residues) exhibit high affinity for specific receptors on the cell surface. The target receptor cell surface are mainly plasma membrane-bound proteins that show tyrosine kinase activity. Example of growth factors include granulocyte-macrophage colony-stimulating factor (GM-CSF), vascular endothelial growth factor (VEGF), epidermal growth factor (EGF) and its receptor (EGFR), and platelet-derived growth factor (PDGF). Furthermore, some hormones that affect the cell growth such as estrogen and progestogens are considered as growth factors. Recent literature has shown that growth factors are essential for various physiological function such as wound healing and cancer among others [86]. Table 3 summarizes some growth factors that are expressed in adipose tissues and their impact on adipogenesis and relation to IR and T2DM.

Among the listed (Table 3) growth factors, EGF receptor (EGFR) and TGF- $\beta$ inhibit adipogenesis, whereas the rest of the growth factors exhibit positive effects on adipogenesis. On the other hand, FGF21 and TGF- $\beta$ induce insulin sensitivity while the rest promote IR.

The suppression of EGFR activity reduces adipogenesis and Akt phosphorylation in adipose-derived stem cells, but only the action of FGFR-1 decreases adipogenesis and Akt phosphorylation, whereas ErbB2 inhibition has the opposite effect. Furthermore, ErbB2-mediated suppression of adipogenesis in adipose-derived stem cells requires EGFR activation [67], whereas the inhibition of EGFR signaling leads to increased longevity in diabetic nephropathy [68]. Obese individuals have higher VEGF-C and -D levels in their blood, which is linked to poorer lipid metrics. Neutralization of VEGF-C in the subcutaneous adipose tissue during the development of obesity improves metabolic indices and IR in mice. It has been revealed that the lymphangiogenic factors VEGF-C and $-\mathrm{D}$ have an unexpected function in the modulation of metabolic syndrome-related adipose tissue inflammation [87]. Increased VEGF-C levels are linked to metabolic degradation and the development of IR. Blocking

Table 3 Growth factors in adipose tissues and their role in adipogenesis and IR

\begin{tabular}{llll}
\hline Growth Factors & Expression in adipose tissue & Effect on adipogenesis & $\begin{array}{l}\text { Relation to IR and } \\
\text { T2DM }\end{array}$ \\
\hline EGFR $(62,63)$ & Subcutaneous adipose tissue & Inhibits adipogenesis & Induces IR \\
VEGF-C $[87,88]$ & Adipose tissue, hepatic lipid & Increases adipogenesis & Induces IR \\
CTGF $[89]$ & Preadipocytes & Increases adipogenesis & Induces IR \\
IGF-I [90,91] & Adipocytes & Increases adipogenesis & Improves IR \\
FGF21 $[92]$ & Subcutaneous adipose tissue & Induces adipogenesis & Enhances insulin sensitivity \\
TGF- $\beta$ [93-95] & White adipose tissue & Inhibits adipogenesis & Induces insulin sensitivity \\
\hline
\end{tabular}


VEGF-C in obese people may be a good way to prevent the onset of IR [88]. Connective tissue growth factor (CTGF) is found in abundance in preadipocytes and its expression is connected to body fat accumulation, as well as skeletal muscle and hepatic IR, with CTGF positive cells predominantly seen in fibrotic areas. The expression of CTGF in adipose tissue decreases in a stepwise manner as weight reduction progressed. In obese persons, elevated CTGF expression is linked to adipose tissue growth, adipose tissue fibrosis, and multi-organ IR [89].

In the pathophysiology of obesity, the growth hormone (GH)/insulin-like growth factor (IGF) system is linked. This system is engaged in the crosstalk between adipose tissue, liver, and pituitary, and both GH and IGF-I have direct effects on adipocyte proliferation and differentiation. This system appears to play a key role in visceral adiposity, and there is a rationale for targeting it in the treatment of visceral obesity induced by $\mathrm{GH}$ deficiency, metabolic syndrome, and lipodystrophies [90]. The increase in IGF-1 and the GH dosage were linked to changes in glucose metabolism following the start of GH therapy. Regardless of pubertal stage, all cases of impaired fasting glycaemia and/or impaired glucose tolerance identified after $\mathrm{GH}$ administration are reversible with dietary intervention and do not progress to diabetes mellitus [91]. Fibroblast growth factor 21 (FGF21) promotes the healthy growth of subcutaneous adipose tissue, which increases systemic insulin sensitivity. In insulin-sensitive obese individuals, serum FGF21 levels correlates with the volume of subcutaneous adipose tissue. Circulating FGF21 causes an increase in M2 macrophage polarization and upregulates adiponectin in subcutaneous adipose tissue. In obesity, increased levels of endogenous FGF21 act as a defensive mechanism against systemic IR [92]. Not only does the transforming growth factor- $\beta$ (TGF- $\beta$ ) signaling pathway plays a function in adipogenesis, but it also plays a role in the development process of IR. TGF- $\beta$ partly reduces adipogenesis via the Smad3-dependent pathway. Smad3 is a complex regulator involved in adipose physiology as well as the etiology of obesity and T2DM, suggesting that it might be utilized to treat obesity and other relevant complications [93-95].

\section{Role of Adipokines in Adipogenesis and IR}

The cytokines that are produced by the adipose tissue are called adipokines. Adipokines such as leptin, adiponectin, resistin and chemokine $(\mathrm{C}-\mathrm{C}$ motif) ligand 2 can affect the insulin function and metabolism of lipids and glucose. Adipokines also have influence on the secretion of some hormones and chemokines. Adipose tissue expansion can lead to imbalance of adipokines, and this imbalance can lead to IR, metabolic syndrome, T2DM and cardiovascular disease. However, each adipokine has a different effect on the obesity and development of IR [96]. Table 4 summarizes adipokines in adipose tissues and their roles in adipogenesis and IR.

Among the listed adipokines, MCPIP1 and progranulin induce IR, whereas the remaining adipokines (Table 4) were shown to increase insulin sensitivity. Moreover, only MCPIP1 was shown to impair adipogenesis whereas the other listed adipokines exhibit enhancing effects on adipogenesis. Leptin is an adipokine produced by white adipose tissue in proportion to the size of fat depots. Leptin reduces body fat by suppressing appetite and raising energy expenditure. Leptin has an indirect effect on metabolism by altering sympathetic nervous

Table 4 Adipokines in adipose tissues and their role in adipogenesis and IR

\begin{tabular}{|c|c|c|c|}
\hline Adipokines & Expression in adipose tissue & Effect on adipogenesis & $\begin{array}{l}\text { Relation to IR and } \\
\text { T2DM }\end{array}$ \\
\hline Leptin [97] & Adipocytes & Suppresses adipocytes proliferation & Induces insulin sensitivity \\
\hline Omentin [98] & $\begin{array}{l}\text { Stromal vascular fraction (SVF) of visceral } \\
\text { adipose tissue }\end{array}$ & Induce adipogenesis & Regulates insulin sensitivity \\
\hline Adiponectin [99] & Adipose tissue and endothelial cells & Enhances adipogenesis & Insulin sensitizing effect \\
\hline Vaspin [100] & Visceral \& subcutaneous adipose tissue & Enhances adipogenesis & Insulin sensitizing effect \\
\hline Apelin $[101,102]$ & Adipose tissues and endothelial cells & Enhances adipogenesis & Increase insulin sensitivity \\
\hline MCPIP1[103] & Immune cells (macrophages $\mid$ monocytes) & Impair adipogenesis & Induces IR \\
\hline Progranulin [104, 105] & Adipose tissue, epithelial cells & Not documented & $\begin{array}{l}\text { High levels correlate with } \\
\text { IR }\end{array}$ \\
\hline
\end{tabular}


system activity or insulin sensitivity [97]. It affects adiposity by decreasing cell proliferation in white fat cells by generating inhibitory circulatory factors and contributing to sympathetic tone, both of which restrict cell growth. The stromal-vascular portion of visceral adipose tissue produces omentin. In adults and adolescents, obesity lowers omentin serum concentrations and adipose tissue secretion [98]. Although this adipokine is believed to control insulin sensitivity, its clinical significance requires further investigation.

Adiponectin is the most available peptide produced by adipocytes, and its deficit has been linked to

\section{A}

\section{Adipogenesis}

Insulin Resistance

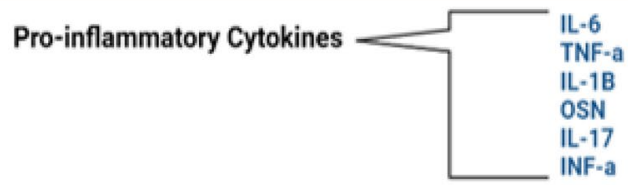

Anti-inflammatory Cytokines
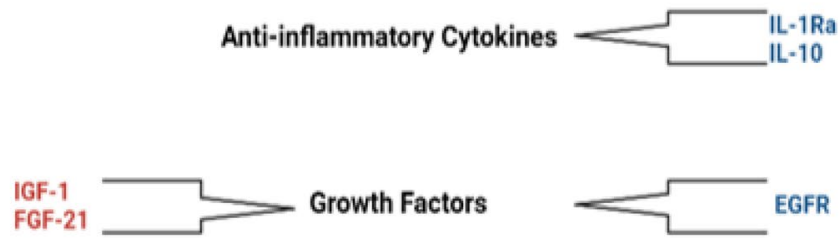

Omentin

Adiponectine

Vaspin

Aplin

Adipokins

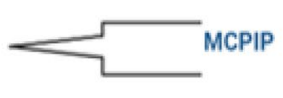

B

\section{Adipogenesis}

\section{Insulin Resistance}
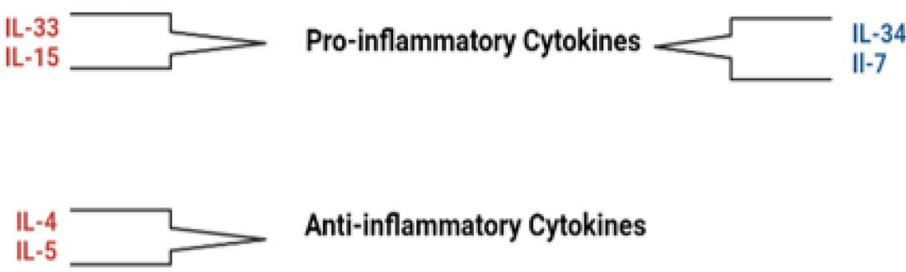

TGF-B

Growth Factors

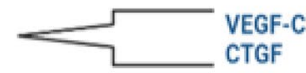

Leptin

Adipokins

Fig. 4 Role of cytokines, growth factors and adipokines in adipogenesis and insulin resistance. The graph shows factors affecting adipogenesis and insulin resistance either in an opposite $\mathbf{A}$ or a similar $\mathbf{B}$ direction. 
obesity-related disorders such IR, T2DM, and cardiovascular disease. Apart from adipocytes, this adipocytokine can be produced by a variety of cells, including skeletal and cardiac myocytes, as well as endothelial cells. Adiponectin's activities are mediated by adiponectin receptors AdipoR1 and AdipoR2. Adiponectin was suggested to protect against IR, diabetes, and atherosclerosis [99]. Vaspin (serpinA12) expression is positively linked with BMI and insulin sensitivity, and it improves glucose tolerance in vivo, suggesting a compensatory function in response to reduced insulin signaling in obesity [100].

Apelin is an adipocyte-produced hormone that plays an important role in energy metabolism. Through the PI3K/Akt and AMPK signaling pathways, apelinAPJ signaling promotes brown adipocyte development by boosting the production of brown adipogenic and thermogenic transcriptional factors. TNF- $\alpha$ suppression of brown adipogenesis is relieved by apelin. Adipocytes' baseline activity is also boosted by apelin. Apelin is able to increase the brown-like characteristics in white adipocytes. The brown adipogenic and browning effects of apelin suggests a potential therapeutic route to combat obesity and related metabolic disorders. Apelin improves not only brown adipocyte differentiation and metabolic activity, but also white adipocyte browning. Apelin-APJ signaling increases browning of adipose tissue [101, 102].

Monocyte chemoattractant protein-1 induced protein1 (MCPIP1) is an RNase that reduces the stability of transcripts that code for inflammatory proteins. MCPIP1 also plays a function in the control of adipogenesis in vitro by lowering the expression of critical transcription factors such as C/EBP $\beta$. Recent studies have shown that MCPIP1 is an essential adipogenesis and adipocyte metabolism regulator [103]. The levels of circulating progranulin were related to BMI, HbA1c, IL-6, and TG in distinct manners. Recent data has indicated that T2DM patients and obese individuals have higher plasma progranulin levels, which is associated with glycolipid metabolism, chronic inflammation, and IR [104, 105]. Progranulin gene expression is elevated during adipocyte development and controlled by multiple inflammatory and metabolic stimuli in a gender, location, and cellspecific dependent manners.

\section{CONCLUSION}

This article reviews some of the most studied factors related to obesity-associated inflammatory response and insulin resistance, which play a critical role in the metabolic consequences of obesity. Various cells within the adipose tissue can secrete specific cytokines, adipokines and growth factors that cause dysfunction of the adipose tissue and impairment of insulin signalling (Fig. 4). The inflammatory environment associated with obesity triggers various inflammatory cascades of adipose tissues through activating specific kinases, which mediate these events. These endocrine and paracrine inflammatory cues within the adipose tissue can further trigger a systemic insulin resistant, inflammatory, and metabolic dyslipidaemia, causing increased risk of T2DM. Understanding these molecular events associated with reduced adipogenesis in insulin resistant obese individuals could help identifying novel therapeutic targets for individuals at higher risk of IR and T2DM.

\section{ACKNOWLEDGMENTS}

Researchers would like to thank Qatar National Research Fund for funding this study.

\section{AUTHORS' CONTRIBUTIONS}

All authors have contributed to reviewing literature, writing the manuscript, reviewing and approving the final version of the manuscript.

\section{FUNDING}

This study was funded by Qatar National Research Fund (UREP26-038-3-015).

\section{AVAILABILITY OF DATA AND MATERIALS}

Not applicable.

\section{DECLARATIONS}

Ethics Approval and Consent to Participate Not applicable.

Competing Interests The authors declare that the research was conducted in the absence of any commercial or financial relationships that could be construed as a potential conflict of interest.

Consent for Publication Not applicable. 


\section{REFERENCES}

1. Wild, S., G. Roglic, A. Green, R. Sicree, and H. King. 2004. Global prevalence of diabetes: Estimates for the year 2000 and projections for 2030. Diabetes Care 27: 1047-1053.

2. Roglic, G. 2016. World Health Organization: Global report on diabetes. Geneva, Switzerland: World Health Organization.

3. Ali, A.T., W.E. Hochfeld, R. Myburgh, and M.S. Pepper. 2013. Adipocyte and adipogenesis. European Journal of Cell Biology 92: 229-236.

4. Hogan, P., T. Dall, P. Nikolov, and A. American Diabetes. 2003. Economic costs of diabetes in the US in 2002. Diabetes Care 26: 917-932.

5. Blair, M. 2016. Diabetes Mellitus Review. Urologic Nursing 36: $27-36$.

6. Clinical guidelines on the identification, evaluation, and treatment of overweight and obesity in adults: executive summary. 1998. Expert Panel on the Identification, Evaluation, and Treatment of Overweight in Adults. American Journal of the Clinical Nutrients 68:899-917.

7. Jaganjac, M., S. Almuraikhy, F. Al-Khelaifi, M. Al-Jaber, M. Bashah, N.A. Mazloum, K. Zarkovic, N. Zarkovic, G. Waeg, W. Kafienah, and M.A. Elrayess. 2017. Combined metformin and insulin treatment reverses metabolically impaired omental adipogenesis and accumulation of 4-hydroxynonenal in obese diabetic patients. Redox Biology 12: 483-490.

8. de Luca, C., and J.M. Olefsky. 2008. Inflammation and insulin resistance. FEBS Letters 582: 97-105.

9. Ros Perez, M., and G. Medina-Gomez. 2011. Obesity, adipogenesis and insulin resistance. Endocrinología y Nutrición 58: $360-369$.

10. Gustafson, B., S. Hedjazifar, S. Gogg, A. Hammarstedt, and U. Smith. 2015. Insulin resistance and impaired adipogenesis. Trends in Endocrinology and Metabolism 26: 193-200.

11. Greenberg, A.S., and M.L. McDaniel. 2002. Identifying the links between obesity, insulin resistance and beta-cell function: Potential role of adipocyte-derived cytokines in the pathogenesis of type 2 diabetes. European Journal of Clinical Investigation 32 (Suppl 3): 24-34.

12. Deng, Y., and P.E. Scherer. 2010. Adipokines as novel biomarkers and regulators of the metabolic syndrome. Annals of the New York Academy of Sciences 1212: E1-E19.

13. Rajpathak, S.N., M. He, Q. Sun, R.C. Kaplan, R. Muzumdar, T.E. Rohan, M.J. Gunter, M. Pollak, M. Kim, J.E. Pessin, et al. 2012. Insulin-like growth factor axis and risk of type 2 diabetes in women. Diabetes 61: 2248-2254.

14. Al-Sulaiti, H., I. Diboun, A.S. Domling, and M.A. Elrayess. 2019. Mediators of Impaired Adipogenesis in ObesityAssociated Insulin Resistance and T2DM. Adipose Tissue - An Update. IntechOpen. https://doi.org/10.5772/intechopen.88746.

15. MacDougald, O.A., and S. Mandrup. 2002. Adipogenesis: Forces that tip the scales. Trends in Endocrinology and Metabolism 13: 5-11.

16. Tam, J., D.G. Duda, J.Y. Perentes, R.S. Quadri, D. Fukumura, and R.K. Jain. 2009. Blockade of VEGFR2 and not VEGFR1 can limit diet-induced fat tissue expansion: role of local versus bone marrow-derived endothelial cells. PLoS One 4:e4974.

17. Feve, B. 2005. Adipogenesis: Cellular and molecular aspects. Best Practice \& Research Clinical Endocrinology \& Metabolism 19: 483-499.

18. Rosen, E.D., and B.M. Spiegelman. 2000. Molecular regulation of adipogenesis. Annual Review of Cell and Developmental Biology 16: 145-171.

19. Cinti, S. 2001. The adipose organ: Morphological perspectives of adipose tissues. The Proceedings of the Nutrition Society 60: 319-328.

20. Camp, H.S., D. Ren, and T. Leff. 2002. Adipogenesis and fat-cell function in obesity and diabetes. Trends in Molecular Medicine 8: 442-447.

21. Grant, R.W., and V.D. Dixit. 2015. Adipose tissue as an immunological organ. Obesity (Silver Spring) 23: 512-518.

22. Lefterova, M.I., and M.A. Lazar. 2009. New developments in adipogenesis. Trends in Endocrinology and Metabolism 20: $107-114$.

23. Garten, A., S. Schuster, and W. Kiess. 2012. The insulinlike growth factors in adipogenesis and obesity. Endocrinology and Metabolism Clinics of North America 41 (283295): v-vi.

24. Khalilpourfarshbafi, M., K. Gholami, D.D. Murugan, M.Z. Abdul Sattar, and N.A. Abdullah. 2019. Differential effects of dietary flavonoids on adipogenesis. European Journal of Nutrition 58: 5-25.

25. Guo, S. 2014. Insulin signaling, resistance, and the metabolic syndrome: Insights from mouse models into disease mechanisms. Journal of Endocrinology 220: T1-T23.

26. Petrie, J.R., T.J. Guzik, and R.M. Touyz. 2018. Diabetes, Hypertension, and Cardiovascular Disease: Clinical Insights and Vascular Mechanisms. Canadian Journal of Cardiology 34: 575-584.

27. Bodhini, D., and V. Mohan. 2018. Mediators of insulin resistance \& cardiometabolic risk: Newer insights. Indian Journal of Medical Research 148: 127-129.

28. Kitessa, S.M., and M.Y. Abeywardena. 2016. Lipid-Induced Insulin Resistance in Skeletal Muscle: The Chase for the Culprit Goes from Total Intramuscular Fat to Lipid Intermediates, and Finally to Species of Lipid Intermediates. Nutrients 8.

29. Longo, M., Zatterale, F., Naderi, J., Parrillo, L., Formisano, P., Raciti, G.A., Beguinot, F., and C. Miele. 2019. Adipose Tissue Dysfunction as Determinant of Obesity-Associated Metabolic Complications. International of Journal Molecular Sciences 20.

30. Medzhitov, R. 2008. Origin and physiological roles of inflammation. Nature 454: 428-435.

31. Newton, K., and V.M. Dixit. 2012. Signaling in innate immunity and inflammation. Cold Spring Harb Perspect Biol 4.

32. Almuraikhy, S., W. Kafienah, M. Bashah, I. Diboun, M. Jaganjac, F. Al-Khelaifi, H. Abdesselem, N.A. Mazloum, M. Alsayrafi, V. Mohamed-Ali, and M.A. Elrayess. 2016. Interleukin-6 induces impairment in human subcutaneous adipogenesis in obesity-associated insulin resistance. Diabetologia 59: 2406-2416. 
33. Klover, P.J., A.H. Clementi, and R.A. Mooney. 2005. Interleukin-6 depletion selectively improves hepatic insulin action in obesity. Endocrinology 146: 3417-3427.

34. Bahar, B., J.V. O'Doherty, and T. Sweeney. 2011. A potential role of IL-6 in the chito-oligosaccharide-mediated inhibition of adipogenesis. British Journal of Nutrition 106: 1142-1153.

35. Cawthorn, W.P., F. Heyd, K. Hegyi, and J.K. Sethi. 2007. Tumour necrosis factor-alpha inhibits adipogenesis via a betacatenin/TCF4(TCF7L2)-dependent pathway. Cell Death and Differentiation 14: 1361-1373.

36. Isakson, P., A. Hammarstedt, B. Gustafson, and U. Smith. 2009. Impaired preadipocyte differentiation in human abdominal obesity: Role of Wnt, tumor necrosis factor-alpha, and inflammation. Diabetes 58: 1550-1557.

37. Palacios-Ortega, S., M. Varela-Guruceaga, M. Algarabel, F. Ignacio Milagro, J. Alfredo Martinez, and C. de Miguel. 2015. Effect of TNF-Alpha on Caveolin-1 Expression and Insulin Signaling During Adipocyte Differentiation and in Mature Adipocytes. Cellular Physiology and Biochemistry 36: 1499-1516.

38. Gagnon, A., C. Foster, A. Landry, and A. Sorisky. 2013. The role of interleukin 1beta in the anti-adipogenic action of macrophages on human preadipocytes. Journal of Endocrinology 217: 197-206.

39. Moratal, C., J. Raffort, N. Arrighi, S. Rekima, S. Schaub, C.A. Dechesne, G. Chinetti, and C. Dani. 2018. IL-1beta- and IL4-polarized macrophages have opposite effects on adipogenesis of intramuscular fibro-adipogenic progenitors in humans. Science and Reports 8: 17005.

40. Lagathu, C., L. Yvan-Charvet, J.P. Bastard, M. Maachi, A. Quignard-Boulange, J. Capeau, and M. Caron. 2006. Longterm treatment with interleukin-1beta induces insulin resistance in murine and human adipocytes. Diabetologia 49: 2162-2173.

41. Martinez-Martinez, E., V. Cachofeiro, E. Rousseau, V. Alvarez, L. Calvier, A. Fernandez-Celis, C. Leroy, M. Miana, R. Jurado-Lopez, A.M. Briones, et al. 2015. Interleukin33/ST2 system attenuates aldosterone-induced adipogenesis and inflammation. Molecular and Cellular Endocrinology 411: 20-27.

42. Miller, A.M., D.L. Asquith, A.J. Hueber, L.A. Anderson, W.M. Holmes, A.N. McKenzie, D. Xu, N. Sattar, I.B. McInnes, and F.Y. Liew. 2010. Interleukin-33 induces protective effects in adipose tissue inflammation during obesity in mice. Circulation Research 107: 650-658.

43. Han, J.M., D. Wu, H.C. Denroche, Y. Yao, C.B. Verchere, and M.K. Levings. 2015. IL-33 Reverses an Obesity-Induced Deficit in Visceral Adipose Tissue ST2+ T Regulatory Cells and Ameliorates Adipose Tissue Inflammation and Insulin Resistance. The Journal of Immunology 194: 4777-4783.

44. Yang, Y.S., X.Y. Li, J. Hong, W.Q. Gu, Y.F. Zhang, J. Yang, H.D. Song, J.L. Chen, and G. Ning. 2007. Interleukin-18 enhances glucose uptake in 3T3-L1 adipocytes. Endocrine 32: 297-302.

45. Almendro, V., G. Fuster, E. Ametller, P. Costelli, F. Pilla, S. Busquets, M. Figueras, J.M. Argiles, and F.J. Lopez-Soriano. 2009. Interleukin-15 increases calcineurin expression in 3T3L1 cells: Possible involvement on in vivo adipocyte differentiation. International Journal of Molecular Medicine 24: 453-458.
46. Lacraz, G., V. Rakotoarivelo, S.M. Labbe, M. Vernier, C. Noll, M. Mayhue, J. Stankova, A. Schwertani, G. Grenier, and A. Carpentier et al. 2016. Deficiency of Interleukin-15 Confers Resistance to Obesity by Diminishing Inflammation and Enhancing the Thermogenic Function of Adipose Tissues. PLoS One 11:e0162995.

47. He, D., Z. Jiang, Y. Tian, H. Han, M. Xia, W. Wei, L. Zhang, and J. Chen. 2018. Genetic variants in IL15 promoter affect transcription activity and intramuscular fat deposition in longissimus dorsi muscle of pigs. Animal Genetics 49: 19-28.

48. Chang, E.J., S.K. Lee, Y.S. Song, Y.J. Jang, H.S. Park, J.P. Hong, A.R. Ko, D.Y. Kim, J.H. Kim, Y.J. Lee, and Y.S. Heo. 2014. IL-34 is associated with obesity, chronic inflammation, and insulin resistance. Journal of Clinical Endocrinology and Metabolism 99: E1263-1271.

49. Lee, M., S.J. Song, M.S. Choi, R. Yu, and T. Park. 2015. IL-7 receptor deletion ameliorates diet-induced obesity and insulin resistance in mice. Diabetologia 58: 2361-2370.

50. Lucas, S., S. Taront, C. Magnan, L. Fauconnier, M. Delacre, L. Macia, A. Delanoye, C. Verwaerde, C. Spriet, and P. Saule et al. 2012. Interleukin-7 regulates adipose tissue mass and insulin sensitivity in high-fat diet-fed mice through lymphocytedependent and independent mechanisms. PLoS One 7:e40351.

51. van Asseldonk, E.J., R. Stienstra, T.B. Koenen, L.J. van Tits, L.A. Joosten, C.J. Tack, and M.G. Netea. 2010. The effect of the interleukin- 1 cytokine family members IL-1F6 and IL-1F8 on adipocyte differentiation. Obesity (Silver Spring) 18: 2234-2236.

52. Katagiri, S., H. Makishima, K. Azuma, Y. Nannya, Y. Saitoh, S. Yoshizawa, D. Akahane, H. Fujimoto, Y. Ito, R. Velaga, et al. 2020. Predisposed genomic instability in pre-treatment bone marrow evolves to therapy-related myeloid neoplasms in malignant lymphoma. Haematologica 105: e337-e339.

53. Miyaoka, Y., M. Tanaka, T. Naiki, and A. Miyajima. 2006. Oncostatin $\mathrm{M}$ inhibits adipogenesis through the RAS/ERK and STAT5 signaling pathways. Journal of Biological Chemistry 281: 37913-37920.

54. Sanchez-Infantes, D., U.A. White, C.M. Elks, R.F. Morrison, J.M. Gimble, R.V. Considine, A.W. Ferrante, E. Ravussin, and J.M. Stephens. 2014. Oncostatin $\mathrm{m}$ is produced in adipose tissue and is regulated in conditions of obesity and type 2 diabetes. Journal of Clinical Endocrinology and Metabolism 99: E217-225.

55. Ahmed, M., and S.L. Gaffen. 2013. IL-17 inhibits adipogenesis in part via C/EBPalpha. PPARgamma and Kruppel-like factors. Cytokine 61: 898-905.

56. Zuniga, L.A., W.J. Shen, B. Joyce-Shaikh, E.A. Pyatnova, A.G. Richards, C. Thom, S.M. Andrade, D.J. Cua, F.B. Kraemer, and E.C. Butcher. 2010. IL-17 regulates adipogenesis, glucose homeostasis, and obesity. The Journal of Immunology 185: 6947-6959.

57. Shin, J.H., D.W. Shin, and M. Noh. 2009. Interleukin-17A inhibits adipocyte differentiation in human mesenchymal stem cells and regulates pro-inflammatory responses in adipocytes. Biochemical Pharmacology 77: 1835-1844.

58. Lee, K., S.H. Um, D.K. Rhee, and S. Pyo. 2016. Interferonalpha inhibits adipogenesis via regulation of JAK/STAT1 signaling. Biochimica et Biophysica Acta 1860: 2416-2427. 
59. Ellulu, M.S., I. Patimah, H. Khaza'ai, A. Rahmat, and Y. Abed. 2017. Obesity and inflammation: The linking mechanism and the complications. Archives of Medical Science 13: 851-863.

60. Klover, P.J., T.A. Zimmers, L.G. Koniaris, and R.A. Mooney. 2003. Chronic exposure to interleukin-6 causes hepatic insulin resistance in mice. Diabetes 52: 2784-2789.

61. Janssens, K., H. Slaets, and N. Hellings. 2015. Immunomodulatory properties of the IL- 6 cytokine family in multiple sclerosis. Annals of the New York Academy of Sciences 1351: $52-60$.

62. Copaescu, A., O. Smibert, A. Gibson, E.J. Phillips, and J.A. Trubiano. 2020. The role of IL-6 and other mediators in the cytokine storm associated with SARS-CoV-2 infection. $J$ Allergy Clin Immunol 146:518-534 e511.

63. Cawthorn, W.P., and J.K. Sethi. 2008. TNF-alpha and adipocyte biology. FEBS Letters 582: 117-131.

64. Ballak, D.B., R. Stienstra, C.J. Tack, C.A. Dinarello, and J.A. van Diepen. 2015. IL-1 family members in the pathogenesis and treatment of metabolic disease: Focus on adipose tissue inflammation and insulin resistance. Cytokine 75: 280-290.

65. Han, J.M., D. Wu, H.C. Denroche, Y. Yao, and C.B. Verchere Levings. 2015. MK: IL-33 Reverses an Obesity-Induced Deficit in Visceral Adipose Tissue ST2+ T Regulatory Cells and Ameliorates Adipose Tissue Inflammation and Insulin Resistance. Journal of immunology (Baltimore, Md : 1950) 194:4777-4783.

66. Yasuda, K., K. Nakanishi, and H. Tsutsui. 2019. Interleukin-18 in Health and Disease. International of Journal Molecular Sciences 20.

67. Quinn, L.S., L. Strait-Bodey, B.G. Anderson, J.M. Argiles, and P.J. Havel. 2005. Interleukin-15 stimulates adiponectin secretion by 3T3-L1 adipocytes: Evidence for a skeletal muscle-to-fat signaling pathway. Cell Biology International 29: 449-457.

68. Hang, H., J.L. Bailey, and C.M. Elks. 2019. Oncostatin M Mediates Adipocyte Expression and Secretion of StromalDerived Factor 1. Biology (Basel) 8.

69. Somm, E., P. Cettour-Rose, C. Asensio, A. Charollais, M. Klein, C. Theander-Carrillo, C.E. Juge-Aubry, J.M. Dayer, M.J. Nicklin, P. Meda, et al. 2006. Interleukin-1 receptor antagonist is upregulated during diet-induced obesity and regulates insulin sensitivity in rodents. Diabetologia 49: 387-393.

70. Somm, E., E. Henrichot, A. Pernin, C.E. Juge-Aubry, P. Muzzin, J.M. Dayer, M.J. Nicklin, and C.A. Meier. 2005. Decreased fat mass in interleukin-1 receptor antagonist-deficient mice: Impact on adipogenesis, food intake, and energy expenditure. Diabetes 54: 3503-3509.

71. Chang, Y.H., K.T. Ho, S.H. Lu, C.N. Huang, and M.Y. Shiau. 2012. Regulation of glucose/lipid metabolism and insulin sensitivity by interleukin-4. International Journal of Obesity 36 : 993-998.

72. Wensveen, F.M., S. Valentic, M. Sestan, T. Turk Wensveen, and B. Polic. 2015. The "Big Bang" in obese fat: Events initiating obesity-induced adipose tissue inflammation. European Journal of Immunology 45: 2446-2456.

73. Yang, C.P., M.Y. Shiau, Y.R. Lai, K.T. Ho, C.W. Hsiao, C.J. Chen, Y.L. Lo, and Y.H. Chang. 2018. Interleukin-4 Boosts
Insulin-Induced Energy Deposits by Enhancing Glucose Uptake and Lipogenesis in Hepatocytes. Oxidative Medicine and Cellular Longevity 2018: 6923187.

74. Molofsky, A.B., J.C. Nussbaum, H.E. Liang, S.J. Van Dyken, L.E. Cheng, A. Mohapatra, A. Chawla, and R.M. Locksley. 2013. Innate lymphoid type 2 cells sustain visceral adipose tissue eosinophils and alternatively activated macrophages. Journal of Experimental Medicine 210: 535-549.

75. Johnson, A.M., A. Costanzo, M.G. Gareau, A.M. Armando, O. Quehenberger, J.M. Jameson, and J.M. Olefsky. 2015. High fat diet causes depletion of intestinal eosinophils associated with intestinal permeability. PLoS One 10:e0122195.

76. Acosta, J.R., B. Tavira, I. Douagi, A. Kulyte, P. Arner, M. Ryden, and J. Laurencikiene. 2019. Human-Specific Function of IL-10 in Adipose Tissue Linked to Insulin Resistance. Journal of Clinical Endocrinology and Metabolism 104: 4552-4562.

77. Rajbhandari, P., B.J. Thomas, A.C. Feng, C. Hong, J. Wang, L. Vergnes, T. Sallam, B. Wang, J. Sandhu, and M.M. Seldin et al. 2018. IL-10 Signaling Remodels Adipose Chromatin Architecture to Limit Thermogenesis and Energy Expenditure. Cell 172:218-233 e217.

78. Annamalai, D., and N.A. Clipstone. 2014. Prostaglandin F2alpha inhibits adipogenesis via an autocrine-mediated interleukin-11/glycoprotein 130/STAT1-dependent signaling cascade. Journal of Cellular Biochemistry 115: 1308-1321.

79. Kwon, H., S. Laurent, Y. Tang, H. Zong, P. Vemulapalli, and J.E. Pessin. 2014. Adipocyte-specific IKKbeta signaling suppresses adipose tissue inflammation through an IL13-dependent paracrine feedback pathway. Cell Reports 9: $1574-1583$.

80. Zamani, N., and C.W. Brown. 2011. Emerging roles for the transforming growth factor- $\{$ beta $\}$ superfamily in regulating adiposity and energy expenditure. Endocrine Reviews 32: 387-403.

81. Meier, C.A., E. Bobbioni, C. Gabay, F. AssimacopoulosJeannet, A. Golay, and J.M. Dayer. 2002. IL-1 receptor antagonist serum levels are increased in human obesity: A possible link to the resistance to leptin? Journal of Clinical Endocrinology and Metabolism 87: 1184-1188.

82. Thomas, D., and C. Apovian. 2017. Macrophage functions in lean and obese adipose tissue. Metabolism 72: 120-143.

83. de-Lima-Junior, J.C., G.F. Souza, A. Moura-Assis, R.S. Gaspar, J.M. Gaspar, A.L. Rocha, D.L. Ferrucci, T.I. Lima, S.C. Victorio, and I.L.P. Bonfante et al. 2019. Abnormal brown adipose tissue mitochondrial structure and function in IL10 deficiency. EBioMedicine 39:436-447.

84. Hong, E.G., H.J. Ko, Y.R. Cho, H.J. Kim, Z. Ma, T.Y. Yu, R.H. Friedline, E. Kurt-Jones, R. Finberg, M.A. Fischer, et al. 2009. Interleukin-10 prevents diet-induced insulin resistance by attenuating macrophage and cytokine response in skeletal muscle. Diabetes 58: 2525-2535.

85. Lind, M. 1996. Growth factors: Possible new clinical tools. $A$ review. Acta Acta Orthopaedica Scandinavica 67: 407-417.

86. Stone, W.L., L. Leavitt, M. Varacallo. 2021. Physiology, Growth Factor. In StatPearls. Treasure Island (FL)

87. Karaman, S., M. Hollmen, S.Y. Yoon, H.F. Alkan, K. Alitalo, C. Wolfrum, and M. Detmar. 2016. Transgenic 
overexpression of VEGF-C induces weight gain and insulin resistance in mice. Science and Reports 6: 31566.

88. Karaman, S., M. Hollmen, M.R. Robciuc, A. Alitalo, H. Nurmi, B. Morf, D. Buschle, H.F. Alkan, A.M. Ochsenbein, K. Alitalo, et al. 2015. Blockade of VEGF-C and VEGF-D modulates adipose tissue inflammation and improves metabolic parameters under high-fat diet. Mol Metab 4: 93-105.

89. Yoshino, J., B.W. Patterson, and S. Klein. 2019. Adipose Tissue CTGF Expression is Associated with Adiposity and Insulin Resistance in Humans. Obesity (Silver Spring) 27: 957-962.

90. Lewitt, M.S. 2017. The Role of the Growth Hormone/InsulinLike Growth Factor System in Visceral Adiposity. Biochem Insights 10: 1178626417703995.

91. Witkowska-Sedek, E., D. Labochka, A. StelmaszczykEmmel, A. Majcher, A. Kucharska, M. Sobol, K. Kadziela, and B. Pyrzak. 2018. Evaluation of glucose metabolism in children with growth hormone deficiency during longterm growth hormone treatment. Journal of Physiology Pharmacology 69.

92. Li, H., G. Wu, Q. Fang, M. Zhang, X. Hui, B. Sheng, L. Wu, Y. Bao, P. Li, A. Xu, and W. Jia. 2018. Fibroblast growth factor 21 increases insulin sensitivity through specific expansion of subcutaneous fat. Nature Communications 9: 272.

93. Tsurutani, Y., M. Fujimoto, M. Takemoto, H. Irisuna, M. Koshizaka, S. Onishi, T. Ishikawa, M. Mezawa, P. He, S. Honjo, et al. 2011. The roles of transforming growth factorbeta and Smad3 signaling in adipocyte differentiation and obesity. Biochemical and Biophysical Research Communications 407: 68-73.

94. Inoh, Y., T. Furuno, N. Hirashima, D. Kitamoto, and M. Nakanishi. 2011. Rapid delivery of small interfering RNA by biosurfactant MEL-A-containing liposomes. Biochemical and Biophysical Research Communications 414: 635-640.

95. Tan, C.K., N. Leuenberger, M.J. Tan, Y.W. Yan, Y. Chen, R. Kambadur, W. Wahli, and N.S. Tan. 2011. Smad3 deficiency in mice protects against insulin resistance and obesity induced by a high-fat diet. Diabetes 60: 464-476.

96. Freitas, P., D. Carvalho, A.C. Santos, A.J. Madureira, E. Martinez, J. Pereira, A. Sarmento, and J.L. Medina. 2014. Adipokines, hormones related to body composition, and insulin resistance in HIV fat redistribution syndrome. BMC Infectious Diseases 14: 347.
97. Harris, R.B. 2014. Direct and indirect effects of leptin on adipocyte metabolism. Biochimica et Biophysica Acta 1842: 414-423.

98. Escote, X., S. Gomez-Zorita, M. Lopez-Yoldi, I. MiltonLaskibar, A. Fernandez-Quintela, J.A. Martinez, M.J. Moreno-Aliaga, and M.P. Portillo. 2017. Role of Omentin, Vaspin, Cardiotrophin-1, TWEAK and NOV/CCN3 in Obesity and Diabetes Development. International of Journal Molecular Sciences 18.

99. Achari, A.E., and S.K. Jain. 2017. Adiponectin, a Therapeutic Target for Obesity, Diabetes, and Endothelial Dysfunction. Int J Mol Sci 18.

100. Heiker, J.T. 2014. Vaspin (serpinA12) in obesity, insulin resistance, and inflammation. Journal of Peptide Science 20: 299-306.

101. Gao, C.L., G.L. Liu, S. Liu, X.H. Chen, C.B. Ji, C.M. Zhang, Z.K. Xia, and X.R. Guo. 2011. Overexpression of PGC-1beta improves insulin sensitivity and mitochondrial function in 3T3-L1 adipocytes. Molecular and Cellular Biochemistry 353: $215-223$.

102. Than, A., H.L. He, S.H. Chua, D. Xu, L. Sun, M.K. Leow, and P. Chen. 2015. Apelin Enhances Brown Adipogenesis and Browning of White Adipocytes. Journal of Biological Chemistry 290: 14679-14691.

103. Losko, M., D. Dolicka, N. Pydyn, U. Jankowska, S. KedrackaKrok, M. Kulecka, A. Paziewska, M. Mikula, P. Major, M. Winiarski, et al. 2020. Integrative genomics reveal a role for MCPIP1 in adipogenesis and adipocyte metabolism. Cellular and Molecular Life Sciences 77: 4899-4919.

104. Qu, H., H. Deng, and Z. Hu. 2013. Plasma progranulin concentrations are increased in patients with type 2 diabetes and obesity and correlated with insulin resistance. Mediators Inflammation 2013:360190.

105. Schmid, A., A. Hochberg, A.F. Kreiss, J. Gehl, M. Patz, M. Thomalla, F. Hanses, T. Karrasch, A. Schaffler. 2020. Role of progranulin in adipose tissue innate immunity. Cytokine 125:154796.

Publisher's Note Springer Nature remains neutral with regard to jurisdictional claims in published maps and institutional affiliations. 